\title{
Elastic lists for facet browsing and resource analysis in the enterprise
}

\author{
Moritz Stefaner \\ Interaction Design Lab, University of Applied Sciences Potsdam, Germany \\ moritz.stefaner@fh-potsdam.de \\ Thomas Urban \\ University of Applied Sciences Schmalkalden, Germany \\ t.urban@fh-sm.de \\ Marc Seefelder
SVA BizSphere
marc.seefelder@sva.de
}

\begin{abstract}
Information management and content governance in large corporations is an ongoing challenge. As a case study, we present the adaptation of the elastic lists facet browser for browsing and analysing large resource collections in an intranet of a large telecommunication corporation. The application allows users to explore resource aggregations over multiple classification schemata in parallel, enabling not only personalized information access, but also improved understanding of content distributions, as well as trend analysis and target tracking. We further present empirical usability results of a first prototype from questionnaires and eye tracking studies.
\end{abstract}

\section{Introduction}

We present a case study on the adaptation of the elastic lists principle [14] for the exploration and analysis of large resource collections in the BizSphere content landscape application, which was developed in cooperation with Nortel, a globally acting telecommunication provider.

BizSphere is an integrated web application for corporate information management, communication, and collaboration. Oriented at clients' existing business taxonomies, the information architecture of the system is inherently multidimensional: In addition to taxonomies describing geographic or product related features of a resource, the resource lifecycle status and individual modification events, sales step phases, and related content areas are stored.

Two concepts concerned with resource organization are especially notable:

- Content propagation: content authors can create resources for specific products or countries, but also for whole product groups or global usage. Accordingly, when uploading the resource, it can either be assigned to specific leaf nodes in the respective business hierarchy (e.g., Spain, Germany, and France), or high-level nodes (such as Europe). In the latter case, the content will be propagated downwards, i.e., automatically assigned to the individual countries per default. However, this approach can be overriden by producing a country-specific resource describing the same offering. The same logic holds for the product hierarchy. As a result, the most specific document at a given data point is picked and presented to the user.

- Content nuggets: since business documents frequently need to be updated, a dynamic approach to resource creation allows the ad-hoc creation of documents based on dynamic, nested content blocks named content nuggets. A single nugget constitutes a human readable and machine processable representation of an identifiable piece of business knowledge. It is granularily structured for reuse in single sourcing environments (websites, web applications, or documents) and appropriate for content management processes and automation.

\section{Elastic Lists}

Elastic lists [14] extend traditional user interfaces for facet browsing with the following features (see Figure 1):

- Visualization of weight proportions: Scaling list entries vertically, according to the relative number of resources assigned, provides a preview of predominant and non-dominant metadata values in the current browsing context.

- Emphasis of the characteristic values of a local metadata profile: To understand what makes a data set special compared to the whole collection, elastic 


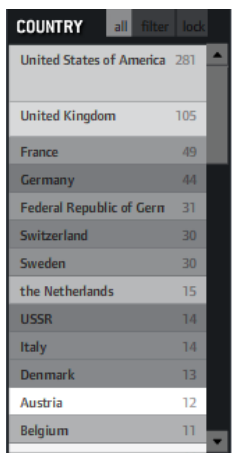

(a)

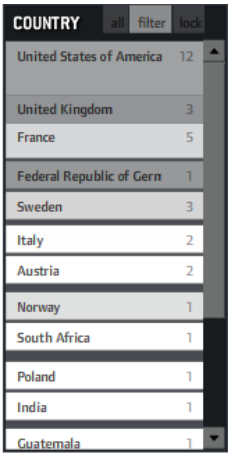

(b)

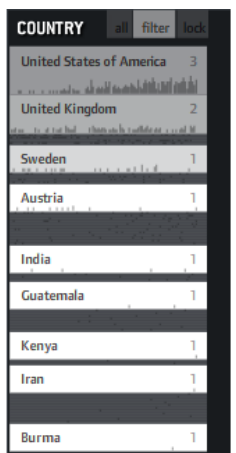

(c)
Figure 1. Different states of an elastic list

lists indicate how the current weights differ from the global distribution through visual weighting by brightness.

- Animated filtering: Animated transitions can facilitate perception of changes in user interface design [5]. Perception of change is especially important for facet browsing, as the sudden disappearance of list items after click can be a source for misconceptions and confusion. In elastic list representation, transitions are animated smoothly and even filtered-out attribute values are still visible as flat lines to make the filtering process more transparent to the user.

Originally described in [14], elastic lists have since been extended to hierarchical, bar chart as well as geo visualization widgets.

\section{Content Landscape}

\subsection{Application overview}

In the BizSphere application, the ContentLandscape module aims at supporting the following user tasks:

- Facilitate content access and browsing: user's interest in resources vary in perspective. For instance, a regional marketing manager for a product group might want to retrieve resources only for his specific region, product group, and restricted to marketing materials. On the other hand, if a presentation for one of his products needs to be localized, he may want to switch to the 'global' regional view, while leaving the rest of the constraints unaltered. Other users may be interested in the latest news across all areas, or material only related to contract preparation. As the content landscape

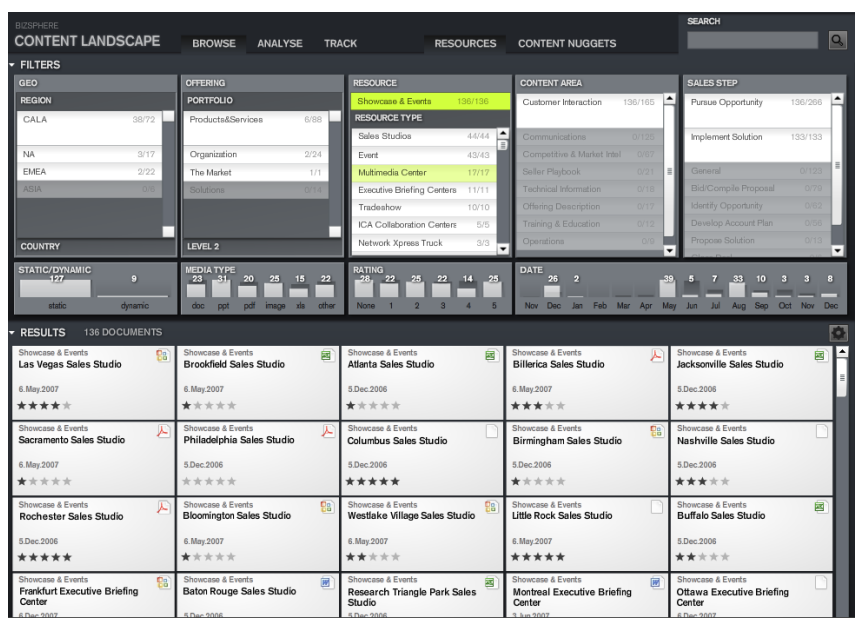

Figure 2. The browsing mode of the content landscape application

application includes all sales resources across departments available in the system, and the possible areas of interest are diverse, a central goal is to allow the user to formulate her personal perspective on the resources in an unrestricted manner.

- Understand content distribution: understanding resource production, use and distribution across departments, regions, and product groups is one of the core challenges of knowledge management in the enterprise [10]. 'What are the most downloaded contents?', 'do the presentation materials for a given product cover all important sales regions?', 'what parts of my resource collection are growing? and which are declining?' are typical questions in this area.

Accordingly, as underlying paradigm for the application, we chose to pursue a faceted browsing and search approach [1] based on dynamic taxonomies [11] as it allows the "rich exploration of a domain across a variety of sources from a user-determined perspective" [6]. The unrestricted exploration of multiple dimensions in parallel supports the desired content access and browsing tasks. Moreover, quantitative analysis of filtered result set statistics with respect to the facet values compared to the overall distributions fosters insight about content distribution and appropriate content governance decisions.

The application consists of two primary modes 'browse' and 'analyse' which are described in the following. 

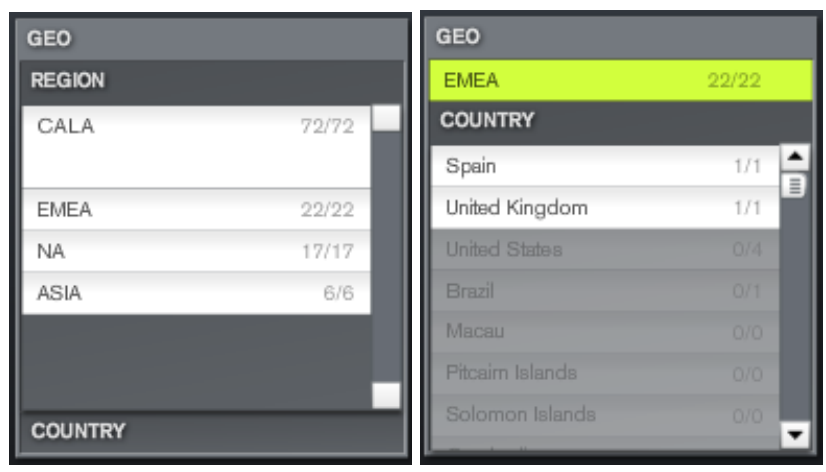

Figure 3. Compact widgets for hierarchical navigation based on elastic lists

\subsection{Browsing mode}

The browsing mode (see Figure 2) allows to select multiple filter settings in flat ('Content Area', 'Sales Step', 'Media type'), hierarchical ('Region', 'Offering' and 'Resource type') and numerical facets ('Rating' and 'Date created'). Matching resources are presented as tiles or lists. The result set is sortable and groupable by all available facets. A detail view for selected resources is available through selection by mouse click.

Animated transitions play a crucial role in making complex changes in user interfaces visually accessible [5]. Consequently, the application is designed to smoothly interpolate between changes in facet and resource presentation, to avoid the notorious phenomenon of change blindness [9] and give users the chance to understand the basic nature of an operation. Moreover, joy of use is an important factor in usability [7], and facilitated by an elegant behavior of the application.

One particular user interface design challenge was to fit deep hierarchies within the contrained space of the filter bar. We chose to use a compact, hierarchical widget based on the accordion pattern ${ }^{1}$, where each hierarchy level is represented as an individual accordion level. On selection of a value, the respective level is collapsed, and the subsequent level opened, to allow further drill-down in the hierarchy. Moreover, opening a level is possible by simply clicking the respective accordion header (see Figure 3). First user evaluations show that this principle is well understood by users (see Section 4).

\footnotetext{
${ }^{1}$ See e.g.,

http://www.welie.com/patterns/showPattern.php?patternID=accordion
}

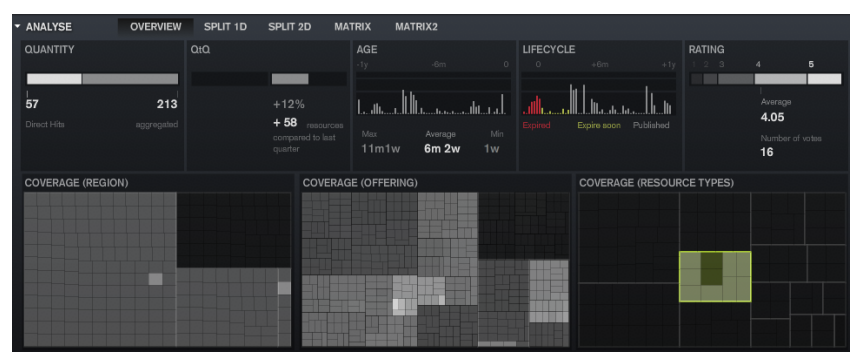

Figure 4. Content analysis dashboard

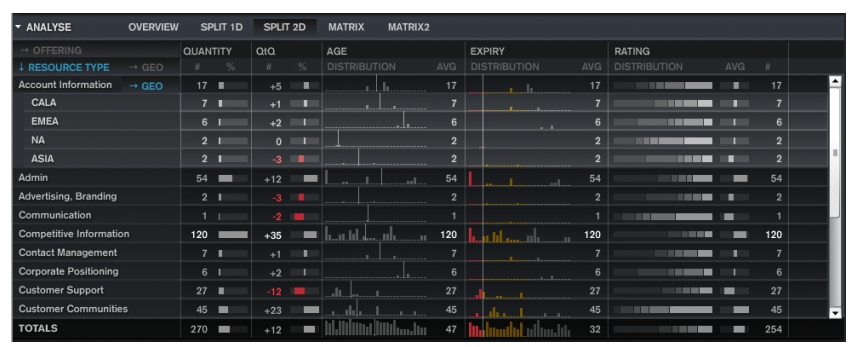

Figure 5. Matrix views for advanced content analytics

\subsection{Resource analysis}

In analysis mode, the same filters are available. However, the content presentation area presents statistical measures about the resource set in the current selection (see Figure 4). It features visualizations of trend measures such as the quarter to quarter growth, a detailed age histogram, and the rating distribution. Moreover, the coverage of the selected resource set with respect to the three main taxonomies 'region', 'offering' and 'resource type' is presented in squarified treemaps $[2,13]$. At first glance, this visual display allows to see, for instance, if all product groups are represented by resources in the current selection, and in which specificity. This statistical analysis can be further decomposed into in several matrix views (see e.g., Figure 5). Inspired by OLAP approaches [3], these matrix views allow the user to split up the result set according to up to 3 dimensions, and compare the statistical measures for the resulting sub-collections in parallel.

\section{Design process and evaluation}

Following a requirements analysis phase, resulting in the definition of scenarios and use cases, the application was prototyped using Adobe Flash. The prototype is a fully functional standalone application for the core browsing cases and operates on a subset of the actual data set. It 


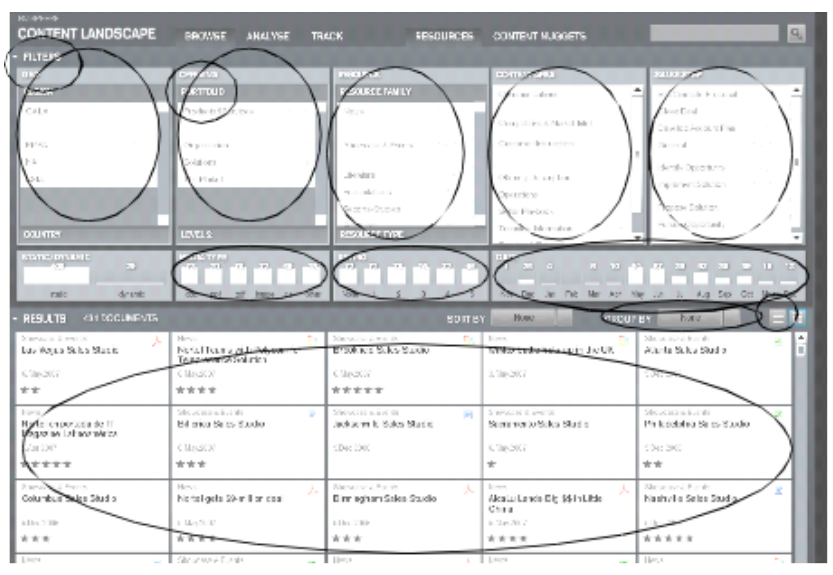

Figure 6. Regions of interest for the eye tracking study

was used for early evaluation of the functionality and interface design (see the following section). Currently, the results of the evaluation are integrated and the application is being developed using Adobe Flex ${ }^{2}$.

\subsection{Experimental design}

We evaluated the usability of the first prototype of the application in an eyetracking study. Eyetracking is the process of measuring the point of gaze in relation to an object of interest. In gaze analysis, the fixation duration is one of the most frequently used parameters. A change of fixation occurs about 3 times per second in quick eye movements, called saccades. It is a common assumption in usability evaluation that the average fixation duration is a correlate of the duration of the respective cognitive processes for understanding and decision $[8,12]$.

The evaluation of the ContentLandscape application was conducted with eleven subjects on various tasks and questions. All subjects were unacquainted with the application and no special introduction or explanation of the application functionalities was given before the experiment.

As a pre-test, we conducted a connect-the-numbers task to measure individual cognitive performance and celerity. These were later correlated with the task performance times. We recorded and analysed the eye movements during the completion of the tasks. Further, we made a video recording of each subject's activities and the computer screen during the experiment.

\footnotetext{
${ }^{2}$ http://www.adobe.com/products/flex/
}

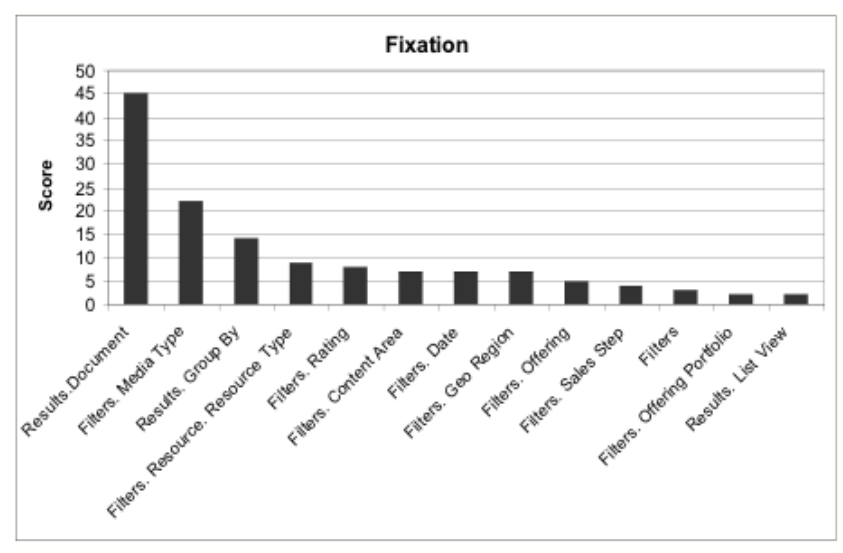

Figure 7. Fixation histogram for the task 'Find all news that are available as a .pdf file'

\subsection{Data analysis}

We analyzed the empirical data for cognitive fixation times, user clicks for task completion, and the time required for both. According to Galley [4], fixation times between 150 and $900 \mathrm{~ms}$ are of special relevance for analysis. These cognitive fixations were extracted from the eye tracking data to define regions of interest in the application (see Figure 6). For instance, for the task 'Find all news that are available as a .pdf', a histogram (see Figure 7) shows the centers of attention for this specific task. The histogram is based on a linearly decreasing scoring model, which emphasizes the first against later fixations. Click data for the same task (see Figure 8) reveals that most subjects failed to click the two necessary filters on the first attempt; a common mistake was to click the 'ppt' instead of the 'pdf' button. This suggests modifications in filter arrangement, such as an increased font size, could help improve the application further.

The analysis of the task completion times compared to the connect-the-numbers task revealed a positive correlation in completion times of $73 \%$.

A questionnaire was used for qualitative evaluation. Generally speaking, most users were able to employ the filtering logic for complex analysis and browsing cases without prior exposition to the software. Most notably, all users understood that grayed out list elements correspond to zero hits in the current context. Seventy-five percent of the subjects found the navigation understandable and understood well, which filters were set in different situations.

Anecdotally, the statement 'I would prefer to be guided through the filter-options step by step' was controversial among the users, resulting in an almost even, homogenous 


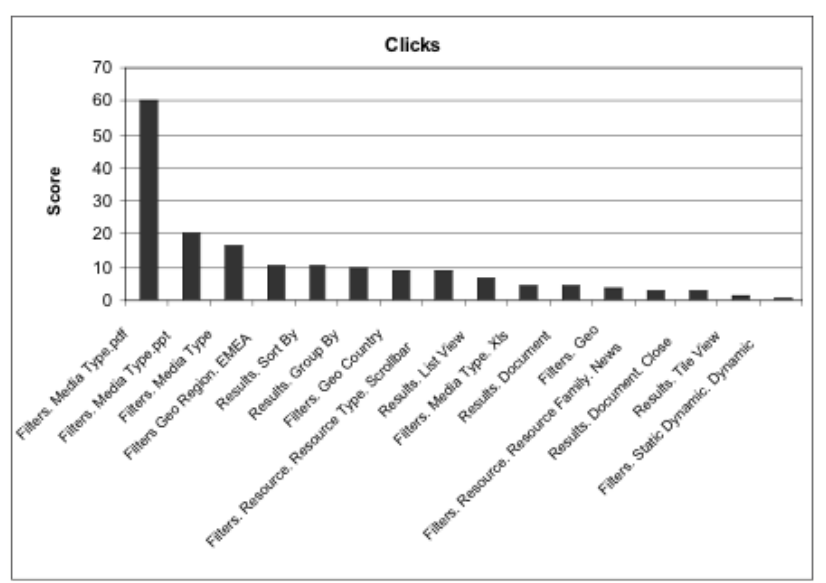

Figure 8. Click histogram

distribution ranging from full agreement over indifference to disagreement. Apparently, although the facet browsing paradigm was generally understood well, some users would have preferred guided navigation for fulfilling their tasks, while others appreciate the unrestricted nature of the interface.

\section{Conclusion and outlook}

We presented a case study on the integration of the elastic lists principle for facet browsing into an enterprise application. Although the application is still under development, initial evaluation results already reveal a high degree of acceptance among users. In the BizSphere environment, facet browsing presents a addition to the existing traditional search and browsing interfaces. We plan to make similar mechanisms available for browsing and understanding the re-use of structured dynamic content snippets (called 'content nuggets') and extend the matrix views and dashboard towards more advanced analysis cases.

\section{References}

[1] R. B. Allen. Retrieval from facet spaces. Electronic Publishing, 8:247-257, 1995.

[2] M. Bruls, K. Huizing, and J. van Wijk. Squarified treemaps. In Proc. of Joint Eurographics and IEEE TCVG Symp. on Visualization (TCVG 2000), pages 33-42. IEEE Press, 2000.

[3] E. F. Codd, S. B. Codd, and C. T. Salley. Providing OLAP (On-Line Analytical Processing) to User-Analysis: An IT Mandate, 1993. White paper.

[4] N. Galley and L. Galley. Saccadic latency and fixation durations as indicators of mental speed. Personality Psychology in Europe, 7:221-234, 1999.
[5] J. Heer and G. Robertson. Animated transitions in statistical data graphics. IEEE Transactions on Visualization and Computer Graphics, 13(6):1240-1247, 2007.

[6] D. Karger and M. Schraefel. The pathetic fallacy of rdf, http://swui.semanticweb.org/swui06/papers/karger /pathetic_fallacy.html, 2006.

[7] D. A. Norman. Emotional Design: Why We Love (Or Hate) Everyday Things. Basic Books, January 2004.

[8] S. Pannasch. Ereignisbezogene Veränderungen der visuellen Fixationsdauer. PhD thesis, TU Dresden, 2003.

[9] J. O. R. Rensink and J. Clark. To see or not to see: The need for attention to perceive changes in scenes. Psychological Science, (8):368-373, 1997.

[10] A. Rockley. Managing Enterprise Content: A Unified Content Strategy. New Riders Press, October 2002.

[11] G. M. Sacco. Dynamic taxonomies: A model for large information bases. IEEE Trans. Knowl. Data Eng., 12(2):468479, 2000.

[12] K. Schiersch. Betrachtungen von Entscheidungsprozessen mittels Blickbewegungsanalyse. Verlag Dr. Kovac, 1997.

[13] B. Shneiderman. Tree visualization with tree-maps: 2d space-filling approach. ACM Transactions on Graphics (TOG), 11(1):92-99, 1992.

[14] M. Stefaner and B. Müller. Elastic lists for facet browsers. In DEXA '07: Proceedings of the 18th International Conference on Database and Expert Systems Applications, pages 217-221, Washington, DC, USA, 2007. IEEE Computer Society. 